\title{
Development and evaluation of a new fully automatic motion detection and correction technique in cardiac SPECT imaging
}

\author{
Chuanyong Bai, PhD, a Jamshid Maddahi, MD, , Joel Kindem, PhD, a \\ Richard Conwell, BS, ${ }^{a}$ Michael Gurley, BS, ${ }^{a}$ and Rex Old, CNMT ${ }^{\mathrm{a}}$
}

Background. In cardiac SPECT perfusion imaging, motion correction of the data is critical to the minimization of motion introduced artifacts in the reconstructed images. Software-based (data-driven) motion correction techniques are the most convenient and economical approaches to fulfill this purpose. However, the accuracy is significantly affected by how the data complexities, such as activity overlap, non-uniform tissue attenuation, and noise are handled.

Methods. We developed STASYS, a new, fully automatic technique, for motion detection and correction in cardiac SPECT. We evaluated the performance of STASYS by comparing its effectiveness of motion correcting patient studies with the current industry standard software (Cedars-Sinai MoCo) through blind readings by two readers independently.

Results. For 204 patient studies from multiple clinical sites, the first reader identified (1) 69 studies with medium to large axial motion, of which STASYS perfectly or significantly corrected $86.9 \%$ and MoCo $72.5 \%$; and (2) 20 studies with medium to large lateral motion, of which STASYS perfectly or significantly corrected $80.0 \%$ and MoCo $60.0 \%$. The second reader identified (1) 84 studies with medium to large axial motion, of which STASYS perfectly or significantly corrected 82.2\% and MoCo $76.2 \%$; and (2) 34 studies with medium to large lateral motion, of which STASYS perfectly or significantly corrected 58.9\% and MoCo 50.0\%.

Conclusions. We developed a fully automatic software-based motion correction technique, STASYS, for cardiac SPECT. Clinical studies showed that STASYS was effective and corrected a larger percent of cardiac SPECT studies than the current industrial standard software. (J Nucl Cardiol 2009;16:580-9.)

Key Words: Myocardial perfusion imaging $\cdot$ SPECT $\bullet$ image artifacts $\bullet$ image quality • motion correction

\section{INTRODUCTION}

Cardiac motion is a major cause of image artifacts in myocardial perfusion SPECT imaging. ${ }^{1-3}$ Cardiac motion includes different components, such as cardiac contraction, upward creeping, motion introduced by respiratory motion, and motion introduced by patient body motion during the scans. Different techniques have

From the Digirad Corporation ${ }^{\mathrm{a}}$, Poway, CA; Department of Molecular and Medical Pharmacology (Nuclear Medicine) and Medicine (Cardiology), UCLA - David Geffen School of Medicine ${ }^{\mathrm{b}}$, Los Angeles, CA.

Received for publication Aug 26, 2008; final revision accepted Apr 30, 2009.

Reprint requests: Chuanyong Bai, PhD, Digirad Corporation, 13950

Stowe Drive, Poway, CA 92064; chbai@digirad.com.

$1071-3581 / \$ 34.00$

Copyright $(92009$ The Authors(s). This article is published with open access at Springerlink.com

doi:10.1007/s12350-009-9096-7 been investigated and used to address one or multiple of these motion components, including cardiac gating for cardiac contraction, ${ }^{4}$ respiratory motion gating ${ }^{5}$ and external visual tracking systems ${ }^{6}$ for respiratory motion, data-driven software-based motion correction techniques (through decomposing respiratory motion from body motion $)^{2,7-11}$ for cardiac upward creeping and patient body motion, and hardware-based motion tracking techniques ${ }^{6,12}$ for patient body motion.

Among the different components of the cardiac motion, cardiac contraction and motion introduced by respiratory motion are in general non-linear or non-rigid body motion that cannot be expressed as a simple combination of translation and rotation. Cardiac upward creeping and motion of the heart introduced by body motion are in general rigid-body motion. The focus of this work is to detect and correct rigid body motion of the heart in the acquired projection data for cardiac SPECT imaging. 
Hardware-based motion correction techniques use external hardware to track patient motion during SPECT scans and use the detected motion for motion correction using iterative reconstruction algorithms. The strength of these approaches is that they can track real time motion of the markers, from which patient rigid body motion and potentially respiratory motion can be obtained. ${ }^{6}$ Hardware-based techniques, however, have a number of inherent limitations. They cannot detect upward creeping of the heart. They impose the use of external motion tracking systems and potentially additional requirement of the room setting, as well as increased complexity of imaging procedure. And also, the accuracy is limited by how accurate one can correlate the tracked motion to real cardiac motion for motion correction.

Compared to hardware-based techniques, softwarebased (data-driven) motion correction techniques are more convenient and economical. Starting with the acquired data, these techniques can detect and correct the axial and lateral (transaxial) translation of the data in each projection view. Therefore, they can handle upright creeping of the heart and patient body motion during the scan. Unfortunately, they can only detect translational motion and the accuracy is limited by how they handle the complexity of SPECT data, such as activity overlap, non-uniform tissue attenuation, and noise.

There are numerous software-based motion correction techniques that have been investigated and published for cardiac SPECT imaging. Most of these techniques fall into the following three categories:

(1) Manual motion correction technique: In this technique, users visually assess if the data in a projection view contains patient motion, and if yes, users manually move the data up and down and/or left and right to correct axial or transaxial motion. This approach can be effective but is time consuming and user dependent, thus not practical in clinical applications. However, it can be used in conjunction with other techniques as a supplemental motion correction tool.

(2) Semi-automatic/automatic motion correction techniques using projection data only approaches: These approaches include (a) cross-correlation of summed horizontal and vertical profiles of successive projection views, ${ }^{7}$ (b) linogram/sinogram crosscorrelation approach, ${ }^{8}$ (c) diverging square approach to track the center of the heart in successive projection views, ${ }^{9}$ and (d) two-dimensional fit in which an operator defines a circular region of interest at a particular projection view to track the heart in successive projection views. ${ }^{2}$ Techniques in this category are not reliable for clinical applications with poor statistics, non-uniform tissue attenuation, and activity overlap of the heart with other organs such as liver.

(3) Automatic motion correction techniques using the projection/reprojection fitting approach: These techniques use sinogram consistency ${ }^{10}$ that assumes the reprojected data is motion free and fits the acquired projection data to the reprojected data to detect motion. The fitting is performed iteratively, assuming the corrected data gets closer to true sinogram data after subsequent iteration. Matsumoto et $\mathrm{al}^{11}$ proposed an extension to the sinogram approach that computes and matches the gradients in the projection and reprojection data with additional weights given to regions corresponding to the myocardium in the reconstructed images. This approach is so far the most successful motion correction solution. It is widely used in the industry as the de facto standard, software-based motion correction package and is known as Cedars-Sinai MoCo (Cedars-Sinai Medical Center, Artificial Intelligence in Medicine (AIM) Los Angeles, CA 90048, referred to as MoCo in the rest of the article).

In this work we implemented and evaluated STASYS, a fully automatic software-based motion correction technique for cardiac perfusion SPECT. The fundamental challenge to software-based motion correction techniques is the complexity of cardiac SPECT data, namely, activity overlap, non-uniform attenuation, and noise. STASYS uses a series of approaches to handle the data complexity, including filtering of the projection data to be motion corrected and the filtering of the reconstructed image before the reprojection, etc. In the rest of this article, we first briefly explain how STASYS works for motion detection and correction, then show phantom and clinical data to demonstrate its performance compared to MoCo.

\section{METHODS}

\section{Projection/Reprojection Fitting}

In the projection/reprojection fitting approach, multiple iterations are generally used. In the first iteration, we reconstruct a three-dimensional volume from the raw projection (data to be motion corrected) using a filtered backprojection algorithm (FBP). We then reproject the volume to generate a reprojection data. The reprojection uses the projector described in Ref. 13 with modeling of the data acquisition geometry. During this FBP reconstruction and reprojection procedure, the motion in the raw projection data is averaged over all the projection views, so the reprojection data can be treated as motion-free data. We then fit the raw projection data to the reprojection data to extract the motion in the raw projection 
data view-by-view. The raw projection data is then motion corrected.

In the second iteration, the motion corrected raw data from the first iteration is used as the projection data to be motion corrected. The detected motion in this iteration is added to that from the first iteration view-by-view to obtain the summed detected motion. The raw projection data is then motion corrected using the summed detected motion.

If the projection/reprojection fitting is correct, each iteration incrementally corrects the motion in the raw projection data until the correction converges, i.e., the data become motion-free. In reality, however, due to the data complexity in cardiac perfusion SPECT, the projection/reprojection fitting approach may not converge, or it may converge but does not converge to the correct results.

In order to make the iterations converge correctly, we first smooth the projection data using a low-pass filter to suppress noise, and then smooth the reconstructed volume before the reprojection step in each iteration. Finally, before the projection/reprojection fitting, (1) we compute the edge patterns in both the projection and reprojection data, followed by (2) segmentation to remove island edges due to noise blobs and (3) normalization of the patterns between the projection and reprojection data to the same maximum in a view-by-view manner.

The use of the edge information and the normalization of the edge patterns between the projection and reprojection data are for the minimization of the effect of the non-uniform attenuation in the projection data, as well as the mismatch of the attenuation information in the projection and reprojection data. ${ }^{14}$ The fitting step is to move the region-of-interest (ROI) of the reprojection data relative to projection data in both axial and transaxial directions to compute the total squared difference (TSD) of all the pixels in the ROI. At each frame, the edge information in the ROI of the projection and reprojection data is normalized to the same maximum. The motion vector that results in the least TSD in the ROI is deemed as the detected motion of the frame. The detected motion is later used to motion correct the original projection data.

The identification of the ROI for fitting is patient specific and fully automatic. All the associated computation mentioned above, such as computation of the edge information and the normalization, are fully automatic as well. These make STASYS an approach that is fully automatic and requires no user interaction.

\section{Evaluation: Phantom Studies}

For the baseline evaluation of the performance of STASYS, we first performed physical phantom studies using a cardiac phantom in air, i.e., only the cardiac insert of the anthropomorphic phantom (Data Spectrum, Hillsborough, $\mathrm{NC}$ ). The myocardium wall of the cardiac insert was injected with $11.1 \mathrm{MBq}$ of Tc-99m. The acquisition was performed in $180^{\circ}$ with 64 projection steps and 10 seconds per step. The radius of the rotation was $28.5 \mathrm{~cm}$. The pixel size of the data was $3.25 \mathrm{~mm}$. The phantom was moved during the acquisition both axially and transaxially. In one study, only slight motion was introduced, i.e., one small move within $1.5 \mathrm{~cm}$ in about every six projection steps with about $2.0 \mathrm{~cm}$ cumulative motion. In a second study, large and frequent motion was introduced, i.e., one move of $1.5 \mathrm{~cm}$ or more in about every three projection steps with over $6.0 \mathrm{~cm}$ cumulative motion.

\section{Evaluation: Patient Studies}

From a large de-identified patient database, a scientist (first author of this paper) identified (1) 110 studies with no visible motion, (2) 110 studies with small or medium motion, and (3) 110 studies with medium or large motion via visual assessment. If the motion in a study appeared to be less than two pixels, the study was identified as with small or medium motion, if the motion appeared to be more than two pixels, the study was categorized as with medium to large motion. The pixel size in these studies was $3.25 \mathrm{~mm}$.

The data in the database were acquired on single, dual, and triple-head Digirad (Digirad Corporation, Poway, CA) cameras from over 10 different clinical sites in the United States. The studies collected from each clinical site were consecutive patient studies acquired during a time period ranging from 1 day to several weeks.

The patient study selection process included two steps. The scientist first ordered the studies using study ID so that from top to bottom of the patient list, the clinical site, acquisition camera, imaging protocol, and scanning date associated with the patient studies were random. Then from top to bottom of the patient studies, the scientist visually assessed the motion in each of the studies and put the studies in a database that was dedicated to this work until the number of studies in each category reached 110. Each patient scan (rest or stress) accounted for one patient study in the dedicated database.

For all the 330 patient studies identified, motion correction was performed on each study using both STASYS and MoCo. MoCo correction of the patient studies was done by the same experienced user of MoCo (first author of this paper). During MoCo correction, the axial limits for correction were adjusted when needed and an elliptical mask was drawn on the transverse image of the center slice of the heart to identify the left ventricle for optimal performance of MoCo, as was recommended by the user's manual of MoCo. STASYS correction of studies was fully automatic, thus a batch process was setup to motion correct all the studies.

After the correction, a cine display page was prepared for each patient study for the evaluation of the motion correction performance of STASYS and MoCo and the comparison of the two. In the page, the first one was always the original (uncorrected) data. The second and third were randomly STASYS corrected or MoCo corrected data. An experienced nuclear cardiologist and an experienced CNMT were asked to evaluate the performance of STASYS and MoCo blindly. Readers were blinded of whether the second cine was from STASYS or MoCo, or vise versa.

During the blind reading, readers first visually identified the amount of motion in the original data (the first one in the cine display page) in both axial and transaxial directions. They then identified the effectiveness of the two motion correction 
techniques by comparing the second and third data to the first one in the cine display page. Each reader did the evaluation independently.

The amount of motion was denoted as: no motion; small motion, i.e., motion is small, no concern of motion artifacts; medium motion, i.e., the amount of motion is significant but does not exceed the limit $(\geq 12.4 \mathrm{~mm})$ specified in ASNC guidelines that requires for a scan to be repeated; ${ }^{15}$ and large motion, i.e., motion is significant and may exceed the limit specified in ASNC guidelines.

The effectiveness of motion correction was scored from 0 to 4 , with 4 perfect correction (PC), 3 significant correction (SC), 2 partial correction, 1 slight correction, and 0 no correction. If artificial motions were introduced to the data by the motion correction technique, the effectiveness was scored -1 .

A direct comparison of the effectiveness of STASYS and MoCo was performed by directly comparing the second data to the third data in the cine display page. If the second data had less motion than the third data, then the second data was considered to be better motion corrected; if the two had the same motion then the two were considered to be equivalent; and if the second data had more motion than the third data, then the second one was considered to have poorer motion correction than the third.

\section{RESULTS}

\section{Phantom Studies}

The cardiac insert in air physical phantom studies illustrate the baseline performance of STASYS when there is low noise, no overlying tissue attenuation, and no activity overlap. Figure 1 shows the sinograms and the summed linograms of the study with slight motion before and after STASYS. A sinogram is a display of a set of horizontal pixel strips taken from each projection view at the same axial location stacked up for the visualization of lateral patient motion. Similarly, a linogram is a display built by abutting a set of vertical pixel strips taken from each projection view at the same lateral location for the visualization of axial patient motion. The summed linogram is the summation of the linograms at all the lateral locations. The cardiac insert was moved slightly and irregularly during the scan.

For the cardiac insert in air phantom study with large motion, four STASYS and MoCo iterations were used. The sinograms and linograms at iterations 1,2, and 4 are shown in Figure 2. STASYS converges faster than MoCo for this study.

Both STASYS and MoCo significantly corrected the motion occurring in between projection views, but neither of them could correct the intra-frame motion, i.e., motion occurring during the acquisition of a projection view. At the views with intra-frame motion both the sinogram and linogram showed spikes and discontinuity.

\section{Patient Studies}

Reading Results. The first reader only finished the evaluation of 204 out of the 330 studies prepared during the day the blind reading was performed. The second reader, who did the blind reading after the first reader, was asked to read the same 204 studies only.
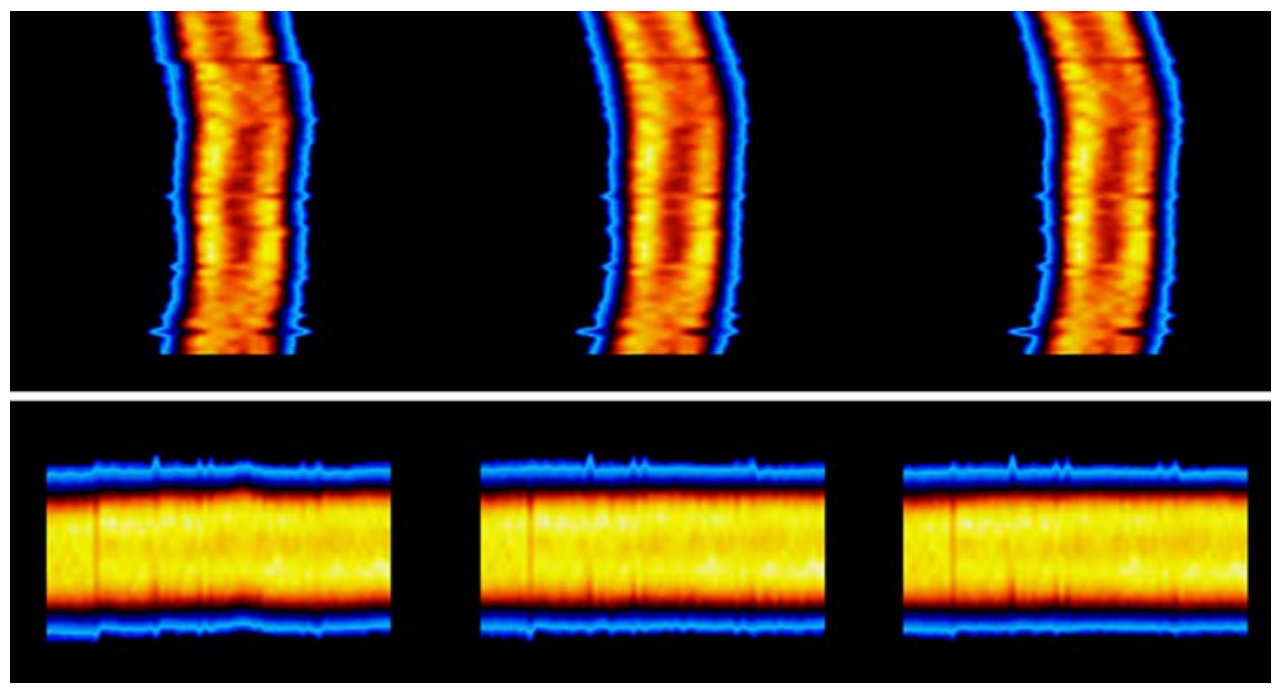

Figure 1. Cardiac insert in air studies with small motions. Top and bottom rows: sinogram and summed linogram of the data. Each row from left to right: before motion correction, with STASYS correction, and with MoCo correction. STASYS and MoCo had similar performance. Neither of the two could correct intra-frame motion. Spikes and discontinuity are visible in the sinogram and linogram at the views with intra-frame motion. 

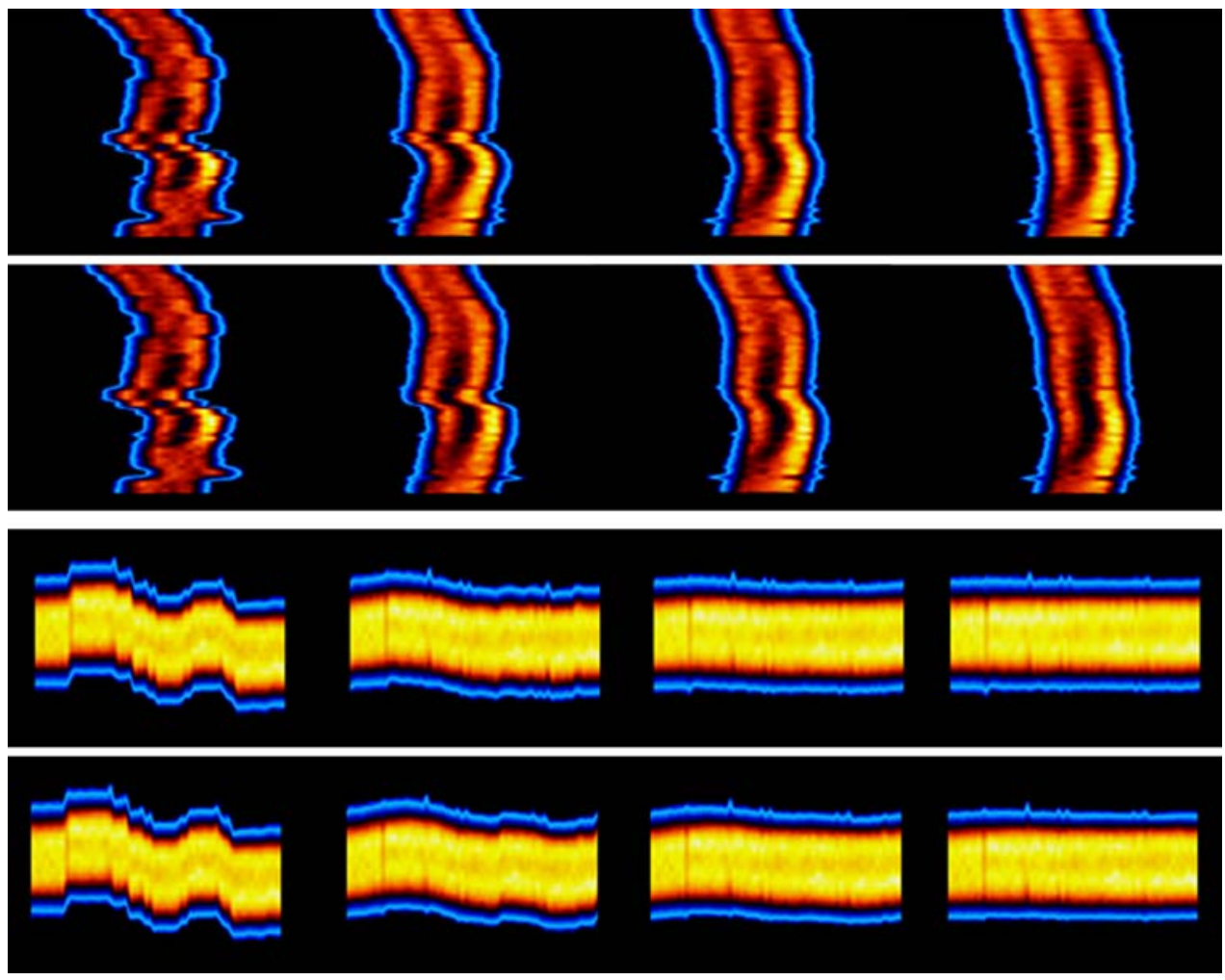

Figure 2. Cardiac insert in air study with large motion. In each row from left to right: before motion correction; with STASYS; or MoCo one iteration, two iterations, and four iterations. The first and third rows: STASYS sinogram and summed linogram, the second and fourth rows: MoCo sinogram and summed linogram. STASYS shows faster convergence than MoCo when correcting the large motion in this study.

After the reading was finished, we analyzed the studies and found that the 204 studies included 72 rest studies using Tl-201 and 132 studies with Tc-99m CardioLite (30 rest and 102 stress studies). Also, out of the 204 studies, 30 were acquired using single-head cameras, 76 using dual-head cameras, and 98 using triple-head cameras.

The first reader identified 62 studies with no motion in the original data. However, the reader identified that in one of these studies, medium axial and transaxial motions were introduced to the data after STASYS correction; and in other two different studies, medium axial motion were introduced into the data after MoCo correction.

For the rest of the studies, the reader identified 131 studies with axial motion and 41 with transaxial motion. Among the studies with axial motion, 62 had small and 69 had medium to large motion. Among the studies with transaxial motion 21 had small and 20 had medium to large motion.

The second reader identified 44 studies with no motion in the original data. Of these studies, the reader identified that medium axial motions was introduced to the data after STASYS correction for the same study that was identified by the first reader; and in a different study medium axial motion was introduced into the data after MoCo correction.

For the rest of the studies, the reader identified 145 with axial motion and 76 with transaxial motion. Among the studies with axial motion, 61 had small and 84 had medium to large motion. Among the studies with transaxial motion 41 had small and 35 had medium to large motion.

Table 1a and $\mathrm{b}$ shows the effectiveness of STASYS and MoCo for correcting all of the studies with axial motion and Table $2 \mathrm{a}$ and $\mathrm{b}$ for only the studies with medium to large axial motion. Table $3 \mathrm{a}$ and $\mathrm{b}$ shows the effectiveness of STASYS and MoCo for correcting all of the studies with transaxial motion and Table $4 a$ and $b$ for only the studies with medium to large transaxial motion. In all these tables, (a) are the results from the first reader and (b) are the results from the second reader.

In general, the results from both the readers demonstrate the performance improvement $(P<0.05$ for studies with medium to large motion) of STASYS over 
Table 1. Effectiveness for correcting all axial motion from the (a) first reader (total 131 studies), (b) second reader (total 145 studies) identified with axial motion

\begin{tabular}{|c|c|c|c|c|c|c|}
\hline Effectiveness & 4 & 3 & $\mathbf{2}$ & 1 & 0 & $<0$ \\
\hline \multicolumn{7}{|l|}{ (a) } \\
\hline STASYS & 105 & 14 & 11 & 0 & 1 & 0 \\
\hline$\%$ of total & 80.2 & 10.7 & 8.4 & 0 & 0.8 & 0 \\
\hline MoCo & 92 & 17 & 16 & 3 & 2 & 1 \\
\hline $\begin{array}{l}\% \text { of total } \\
\text { (b) }\end{array}$ & 70.2 & 13.0 & 12.2 & 2.3 & 1.5 & 0.8 \\
\hline STASYS & 95 & 29 & 18 & 1 & 0 & 2 \\
\hline$\%$ of total & 65.5 & 20.0 & 12.4 & 0.7 & 0.0 & 1.4 \\
\hline MoCo & 89 & 30 & 22 & 1 & 2 & 1 \\
\hline$\%$ of total & 61.4 & 20.7 & 15.2 & 0.7 & 1.4 & 0.7 \\
\hline
\end{tabular}

4: Perfect correction; 3: significant correction; 2: partial correction; 1 : slight correction; 0: no correction; and $<0$ : artificial motion introduced.

Table 2. Effectiveness for correcting medium to large axial motion from the (a) first reader (total of 69 studies), (b) second reader (total of 84 studies) identified with medium to large axial motion. The difference between STASYS and MoCo is significant $(P=0.0003$ for the first reader and 0.0425 for the second reader)

\begin{tabular}{llllllll}
\hline Effectiveness & $\mathbf{4}$ & \multicolumn{1}{c}{$\mathbf{3}$} & $\mathbf{2}$ & $\mathbf{1}$ & $\mathbf{0}$ & $<\mathbf{0}$ \\
\hline $\begin{array}{l}\text { (a) } \\
\text { STASYS }\end{array}$ & 47 & 13 & 9 & 0 & 0 & 0 \\
\% of total & 68.1 & 18.8 & 13 & 0 & 0 & 0 \\
$\begin{array}{l}\text { MoCo } \\
\text { \% of total }\end{array}$ & 34 & 16 & 14 & 3 & 1 & 1 \\
(b) & 49.3 & 23.2 & 20.3 & 4.34 & 1.45 & 1.45 \\
STASYS & 45 & 24 & 13 & 1 & 0 & 1 \\
\% of total & 53.6 & 28.6 & 15.5 & 1.2 & 0 & 1.2 \\
$\begin{array}{l}\text { MoCo } \\
\text { \% of total }\end{array}$ & 39 & 25 & 17 & 1 & 1 & 1 \\
& 46.4 & 29.8 & 20.2 & 1.2 & 1.2 & 1.2 \\
\hline
\end{tabular}

MoCo, even though the improvement is more dramatic as seen by the first than the second reader. To visualize the performance difference between STASYS and MoCo, we plot in Figure 3 the percentage of perfect and significant correction results from the first reader for correcting medium to large motion. The plot illustrates that STASYS is more effective than MoCo in correcting both axial and transaxial motion. Furthermore, the comparison of the results for studies with all motion (Tables 1 and 3) and for studies with only medium to
Table 3. Effectiveness for correcting all transaxial motion from the (a) first reader (total of 41 studies), (b) second reader (total of 76 studies) identified with transaxial motion

\begin{tabular}{llcccll}
\hline Effectiveness & $\mathbf{4}$ & $\mathbf{3}$ & $\mathbf{2}$ & $\mathbf{1}$ & $\mathbf{0}$ & $<\mathbf{0}$ \\
\hline (a) & & & & & & \\
$\quad$ STASYS & 28 & 5 & 7 & 0 & 1 & 0 \\
\% of total & 68.3 & 12.2 & 17.1 & 0 & 2.4 & 0 \\
MoCo & 23 & 8 & 6 & 1 & 3 & 0 \\
$\quad \%$ of total & 56.1 & 19.5 & 14.6 & 2.4 & 7.3 & 0 \\
(b) & & & & & & \\
STASYS & 32 & 15 & 17 & 5 & 6 & 1 \\
\% of total & 42.1 & 19.7 & 22.4 & 6.6 & 7.9 & 1.3 \\
MoCo & 23 & 8 & 6 & 1 & 3 & 0 \\
\% of total & 38.2 & 22.4 & 18.4 & 11.8 & 7.9 & 1.3 \\
\hline
\end{tabular}

Table 4. Effectiveness for correcting medium to large transaxial motion from the (a) first reader (total of 20 studies), (b) second reader (total of 34 studies) identified with medium to large transaxial motion. The difference between STASYS and MoCo is significant $(P=0.0168$ for the first reader and 0.0086 for the second reader)

$\begin{array}{lllllll}\text { Effectiveness } & 4 & 3 & 2 & 1 & 0 & <0\end{array}$

(a)

$\begin{array}{lcccccc}\text { STASYS } & 12 & 4 & 4 & 0 & 0 & 0 \\ \text { \% of total } & 60.0 & 20.0 & 20.0 & 0 & 0 & 0 \\ \text { MoCo } & 8 & 4 & 5 & 1 & 2 & 0 \\ \text { \% of total } & 40.0 & 20.0 & 25.0 & 5.0 & 10.0 & 0\end{array}$

(b)

$\begin{array}{lccccll}\text { STASYS } & 11 & 9 & 10 & 2 & 2 & 0 \\ \text { \% of total } & 32.4 & 26.5 & 29.4 & 5.9 & 5.9 & 0 \\ \text { MoCo } & 8 & 9 & 8 & 5 & 3 & 1 \\ \text { \% of total } & 23.5 & 26.5 & 23.5 & 14.7 & 8.8 & 2.9\end{array}$

large motion (Tables 2 and 4) shows that the improvement in effectiveness of STASYS over MoCo is more significant for studies with medium to large motion $(P<0.05)$ than small motion.

Table 5a and b shows the direct comparison of the effectiveness of STASYS and MoCo for motion correction. STASYS is shown to be superior to MoCo in correcting both axial and transaxial motion, even though the percentage quantitation from the two readers is different. The percentage of studies that STASYS is better 


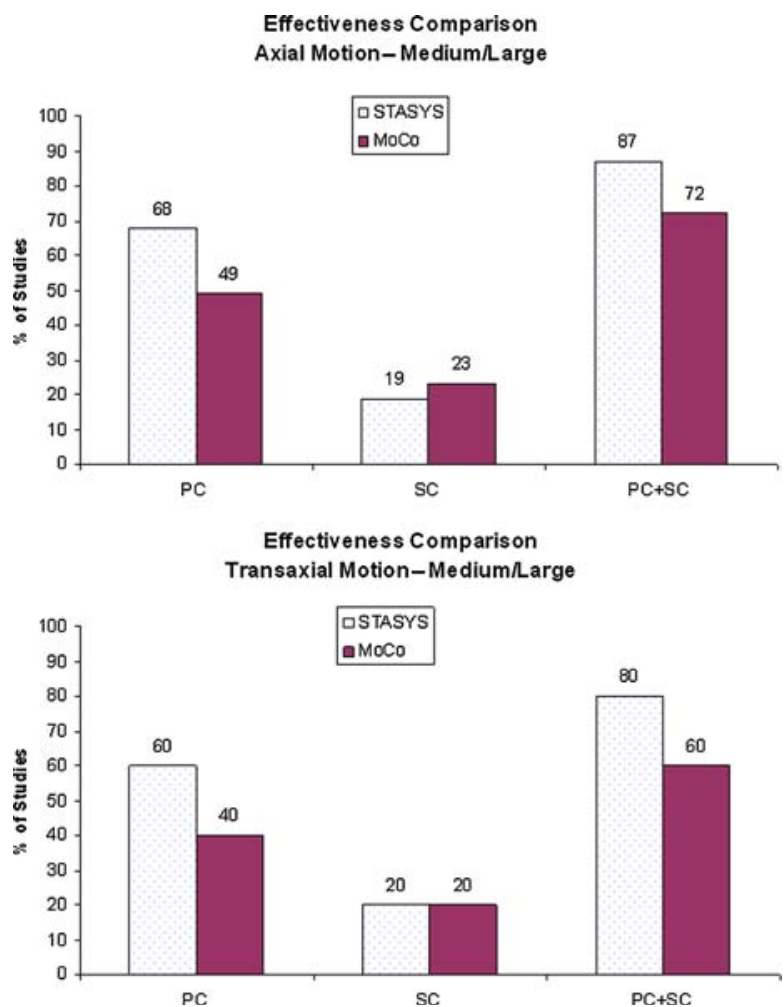

Figure 3. Effectiveness comparison of STASYS and MoCo on correcting medium to large motion (top: axial, bottom: transaxial) from the first reader. $P C$, Perfect correction (score 4); $S C$, significant correction (score 3 ).

than MoCo in correcting motion is higher for patient studies with medium to large motion than for patient studies with small motion, and for both cases, better overall.

Comparing the blind reading results from the two readers, we found that the second reader read slightly more studies with axial motion than the first reader $(145$ out of 204 , i.e., $71.1 \%$ vs 131 out of 204, i.e., $64.2 \%$ ) and significantly more studies with transaxial motion (76 out of 204 , i.e., $37.3 \%$ vs 41 out of 204 , i.e., $20.1 \%$ ). The overall performance difference between STASYS and MoCo seen by the second reader was smaller than that by the first reader.

The percentage of perfect and significant correction $(\mathrm{PC}+\mathrm{SC})$ of axial motion agreed very well between the two readers. For all axial motion, the first reader read $\mathrm{PC}+\mathrm{SC}$ of $90.9 \%$ for STASYS and 83.2 for MoCo, the second reader read $85.5 \%$ and $82.1 \%$, respectively. For axial motion with medium to large motion, the first reader read PC + SC of $86.9 \%$ for STASYS and $72.5 \%$ for $\mathrm{MoCo}$, and the second reader read $82.2 \%$ and $76.2 \%$, respectively.

For transaxial motion correction, the reading results from the second reader differed significantly from those from the first reader. The performance of both STASYS and MoCo on transaxial motion correction was seen significantly lower by the second reader than the first reader. Specifically, for all transaxial motion, the first reader read PC + SC of $80.5 \%$ for STASYS and $75.6 \%$ for $\mathrm{MoCo}$, the second read $61.8 \%$ and $60.6 \%$, respectively. For medium to large transaxial motion, the first reader read $80.0 \%$ for STASYS and $60.0 \%$ for MoCo, and the second reader read $58.9 \%$ and $50.0 \%$, specifically. This difference between the two readers confirmed the common knowledge of the higher level of complexity of transaxial motion detection and correction than axial motion in cardiac SPECT.

Statistical Analysis. For a limited statistic evaluation of the blind reading results, one tail $t$-test showed that the effectiveness difference of STASYS and MoCo on motion correction was significant. For medium to large axial motion, $P$ value was 0.0003 for the first reader and 0.0425 for the second reader; for medium to large transaxial motion, $P$ was 0.0168 for the first reader and 0.0086 for the second reader.

Table 5. Direct comparison of the effectiveness for motion correction from the (a) first reader, (b) second reader (\% of the studies identified with motion)

Axial motion correction

$M C=m C \quad M C>m C \quad M C<m c \quad M C=m c \quad M C>m c \quad M C<m c$

(a)

\begin{tabular}{|c|c|c|c|c|c|c|}
\hline All motion & 84.3 & 12.3 & 3.4 & 94.6 & 4.4 & 1.0 \\
\hline Medium to large motion & 62.3 & 33.3 & 4.3 & 68.2 & 31.8 & 0.0 \\
\hline All motion & 703 & 138 & 60 & 800 & 76 & 34 \\
\hline Medium to large motion & 73.8 & 20.2 & 6.0 & 88.1 & 9.5 & 2.4 \\
\hline
\end{tabular}

$M C=m c$, Equivalent; $M C>m c$, STASYS better than MoCo; and $M C<m c$, STASYS poorer than MoCo. 

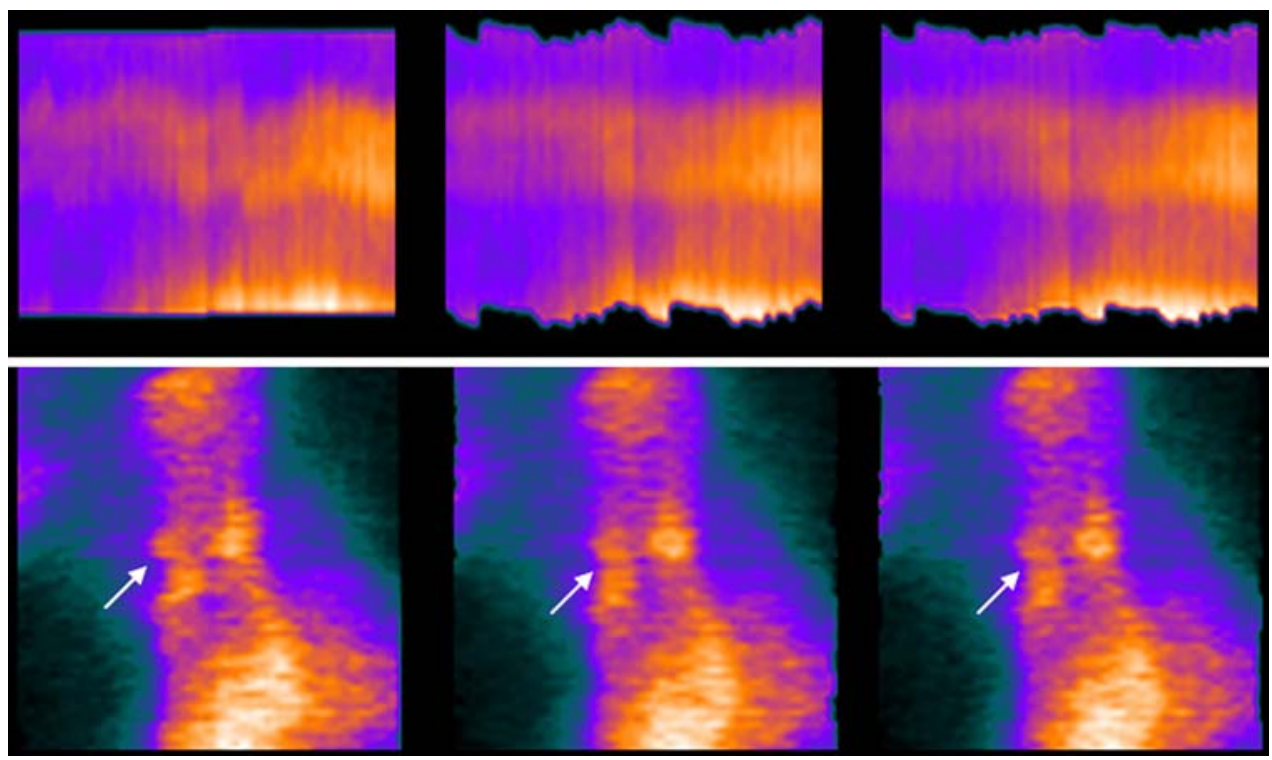

Figure 4. Summed linogram (top left) showed large and frequent axial motions $(\sim 3.6$ pixels, $3.25 \mathrm{~mm} / \mathrm{pixel}$ ) and sinogram (bottom left) showed a medium transaxial motion (1.5 pixels, discontinuity shown by the white arrows) for a patient study acquired on a dual-head camera. Both STASYS (middle) and MoCo (right) showed perfect correction visually, even though the summed linograms showed slightly better correction of STASYS (top middle) than MoCo (top right) in axial direction.
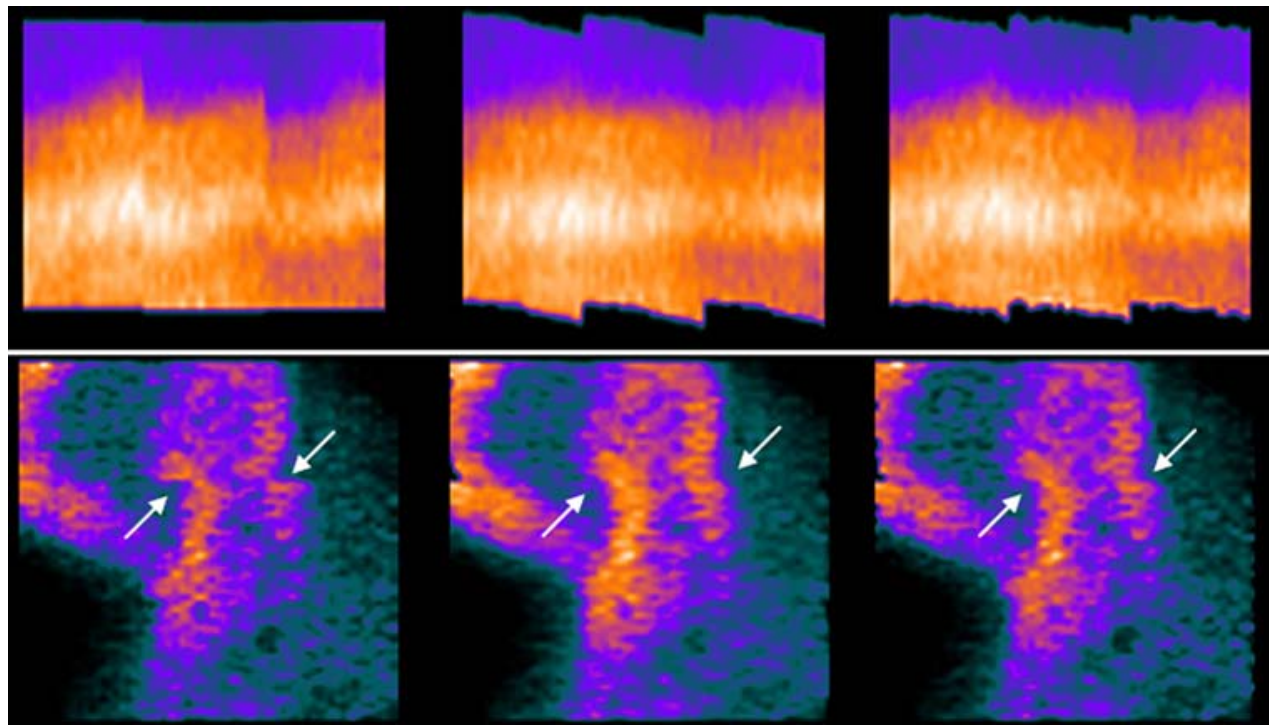

Figure 5. Top Summed linogram (left) showed an axial motion of 3.6 pixels $(3.25 \mathrm{~mm} / \mathrm{pixel})$ of a patient study acquired on a triple-head system. STASYS (middle) showed perfect correction and MoCo (right) showed significant correct for this study. Bottom Sinogram (left) showed a transaxial motion of 3.2 pixels (discontinuity shown by white arrows). STASYS (middle) showed significant correction and MoCo (right) showed partial correction.

Case Examples. As examples of the effect of motion correction of STASYS and MoCo, Figure 4 shows the summed linograms and sinograms of a patient study with large axial and medium transaxial motion. In this study, both
STASYS and MoCo perfectly detect and correct the motion. And Figure 5 shows a study with large axial motion and another study with large transaxial motion. In these studies, STASYS corrects the motion better than MoCo. 


\section{DISCUSSION}

Conventional data acquisition in cardiac SPECT uses histogram mode acquisition such as the data used in this article. In histogram mode acquisition, counts acquired at each frame (frame for non-gated data or each gated bin for gated-data) are added together without distinguishing the time they are detected. Softwarebased motion correction technique cannot, in general, correct for intra-frame motion if the data are acquired in this mode. This is due to the fact that (1) in histogram mode, the portions of the data with and without motion are overlapped when intra-frame motion occurs and cannot be distinguished from each other and (2) software-based correction techniques can only use the data of each frame as a whole. However, if the data are acquired in list-mode, then it is possible that intra-frame motion can be detected, since the time-tag of the data allows for the rebinning of the data at each frame into sub-frames, allowing the distinguishing of the data without motion from data with motion. Therefore, the sub-frames with motion may be identified and corrected.

While STASYS outperformed MoCo by correcting motion in a larger percent of patient studies than MoCo, the overall performance of STASYS still needs to be improved. First of all, STASYS cannot effectively correct (with scores 2 or less) a relative large percent of the studies with transaxial motion. Secondly, the results show that for a small percent of the patient studies MoCo is more effective than STASYS. One potential approach that may improve the performance of STASYS is to use a blend of the edge information and gray information in the projection/backprojection data instead of the edge information alone for the fitting.

STASYS was developed to offer optimized motion correction for use with small field of view dedicated cardiac systems using a cardiocentric imaging acquisition method. In contrast, MoCo was originally developed for use on larger field of view systems using acquisition methods that were not cardiocentric. With new acquisition approaches, the motion correction approach may need to be tailored to the specific acquisition protocol for optimized performance. Images from a small FOV Digirad camera with cardiocentric imaging were used to compare STASYS and MoCo in this work. Whether these results are applicable to large field of view cameras is unknown, and requires further investigation.

\section{CONCLUSION}

We developed a fully automatic software-based motion correction technique, STASYS, for cardiac perfusion SPECT. It uses automatic region identification and the edge information in the projection/reprojection fitting approach to handle activity overlap, non-uniform attenuation, and noise in the projection data. Through blind reading evaluation, STASYS was shown to be effective and correct a larger percent of cardiac SPECT studies than the current industrial standard software (MoCo).

\section{Acknowledgment}

Our many thanks go to Samia Arram (CNMT) for reviewing phantom studies and some patient studies and to Sriranga Agili for helpful discussion during the development of STASYS. We thank Romer De Los Santos and Diana Solis for the development of STASYS software. This work is fully supported by Digirad Corporation (Digirad Corporation, Poway, CA 92064).

\section{Open Access}

This article is distributed under the terms of the Creative Commons Attribution Noncommercial License which permits any noncommercial use, distribution, and reproduction in any medium, provided the original author(s) and source are credited.

\section{References}

1. Friedman J, Van Train K, Maddahi J, Rozanski A, Prigent F, Bietendorf J, et al. Upward creep of the heart: A frequent source of false-positive reversible defects during thallium-201 stress-redistribution SPECT. J Nucl Med 1989;30:1718-22.

2. Cooper JA, Neumann PH, McCandless BK. Effect of patient motion on tomographic myocardial perfusion imaging. J Nucl Med 1992;33:1566-71.

3. Tsui BMW, Segars WP, Lalush DS. Effect of upward creep and respiratory motion in myocardial SPECT. IEEE Trans Nucl Sci 2000;47:1192-5.

4. DePuey E. Artifacts clarified by and caused by gated myocardial perfusion SPECT. In: Germano G, Berman D, editors. Clinical gated cardiac SPECT. Armonk, NY: Futura Publishing; 1999. p. 183-237.

5. Segars WP, Tsui BWM. Study of the efficacy of respiratory gating in myocardial SPECT using the new 4-D NCAT phantom. IEEE Trans Nucl Sci 2002;49:675-9.

6. Bruyant PP, Gennert MA, Speckert GC, Beach RD, Morgenstern JD, Kumar N, et al. A robust visual tracking system for patient motion detection in SPECT: Hardware solutions. IEEE Trans Nucl Sci 2005;52:1288-94.

7. Eisner RL, Noever T, Nowak D, Carlson W, Dunn D, Oates J, et al. Use of cross correlation function to detect patient motion during SPECT imaging. J Nucl Med 1987;28:97-101.

8. Sarkar S, Oghabian MA, Mohammadi I, Mohammadpour A, Rahmim A. A linogram/sinogram cross-correlation method for motion correction in planar and SPECT imaging. IEEE Trans Nucl Sci 2007;54:71-9.

9. Geckle WJ, Frank TL, Links JM, Becker LC. Correction for patient and organ movement in SPECT: Application to exercise thallium-201 cardiac imaging. J Nucl Med 1988;29:441-50.

10. Huang SC, Yu DC. Capability evaluation of a sonogram error detection and correction method in computed tomography. IEEE Trans Nucl Sci 1992;39:1106-10. 
11. Matsumoto N, Berman DS, Kavanagh PB, Gerlach J, Hayes SW, Lewin HC, et al. Quantitative assessment of motion artifacts and validation of a new motion-correction program for myocardial perfusion SPECT. J Nucl Med 2001;42:687-94.

12. Gennert MA, Bruyant PP, Narayanan MV, King MA. Detecting patient motion in SPECT imaging using stereo optical cameras (abstract). J Nucl Med. 2002;43:222P.

13. Bai C, Zeng GL, Grant GT, DiFilippo F, Miller S. Slab-by-slab blurring model for geometric point response correction and attenuation correction using iterative reconstruction algorithms. IEEE Trans Nucl Sci 1998;45:2168-73.

14. Bai C, Conwell RL. Reprojected data from reconstructed volume images cannot replace the acquired raw projection data for the purpose of quality assurance while diagnosing cardiac SPECT studies (abstract). ASNC2008, Boston, USA 2008; 15.36.

15. DePuey EG, Garcia EV. Updated imaging guidelines for nuclear cardiology procedures, Part 1. J Nucl Cardiol 2001;8:G1-58. 